\title{
What influences clinical decision making?
}

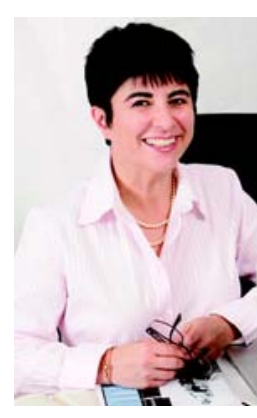

Annette Katelaris Editor akatelaris@mja.com.au doi: 10.5694/mjal2.c0806 s doctors, we spend a lot of time learning facts but little time is formally dedicated to learning how to think. Ideally, this skill is learnt ("picked up") during the years of clinical teaching at the bedside, in the clinic and in the operating theatre. Errors in clinical interpretation and reasoning can occur at any point during patient care. These are often due not to a lack of knowledge or competence, but to the decision-making processes of humans in situations that are clinically complex, uncertain, and pressured by time and emotion.

In this issue, Cruz and colleagues (page 161) looked at the clinical reasoning skills of junior and senior emergency medicine staff by testing their ability to accurately interpret electrocardiographs (ECGs) with no clinical history, a history that was positively biased towards the correct diagnosis, or a history that was negatively biased towards an alternative diagnosis.

Overall, doctors made the correct diagnosis about half of the time. Worryingly, this $52 \%$ correct rate may be the "best-case scenario", as less than half of the doctors approached agreed to participate in the study. One might surmise that these results therefore reflect the doctors who perceive themselves as most competent at interpreting ECGs.

Accuracy was affected by knowing the clinical history and by the seniority of the clinician - results that were to be expected. In reality, however, the pressures associated with the modern emergency department, including overcrowding and the 4-hour rule, make it inevitable that the ECG is usually taken before the patient is seen by a doctor and often interpreted by a junior doctor without the benefit of the history to inform the decision.

The authors did not determine the impact of incorrect diagnoses. If ventricular tachycardia is mistaken for supraventricular tachycardia, the consequences could be severe. Likewise, if a diagnosis of myocardial infarction is made in a patient with pericarditis, it is possible that the patient would undergo invasive, potentially dangerous and unnecessary investigations. In reality, if a doctor is unsure of the diagnosis, one hopes that he or she would be able to consult and collaborate with colleagues about the diagnosis, with knowledge of the patient history and clinical examination.

This study also makes one consider the risks and costs associated with the inappropriate use of investigations to screen for illness and to guide management, particularly if the test has a low sensitivity - like the ECG. For example, many a clinical dilemma has arisen when the result of a Ddimer test, done as part of a workup, is positive for a patient in whom a pulmonary embolism or deep vein thrombosis was not even part of the differential diagnosis.

The statistics about errors in medical reasoning are sobering. The correct diagnosis is missed or delayed in up to $14 \%$ of acute admissions (J Gen Intern Med 2005; 20: 334 339). If the diagnosis is correct, up to $43 \%$ of patients do not receive recommended care (MJA 2012; 197: 100-105), and about $\$ 800$ billion - nearly one-third of all health care spending - is wasted on unnecessary diagnostic tests, procedures and extra days in hospital (http:// www.reuters.com/article/2012/02/16/us-overtreatmentidUSTRE81F0UF20120216). Wilson and colleague's landmark analysis of the cause of adverse events in the Australian health care system reported that almost half of reported adverse events involved errors of reasoning (MJA 1999; 170: 411-415).

Clearly, we overestimate our ability to correctly deploy tests, interpret test results, and act appropriately on the results of clinical interactions and subsequent investigations. Cruz et al's study confirms the need to actively critique our methods of clinical reasoning, and to teach these skills. Formal courses will provide a theoretical background, but the need for this to be included in the curriculum at the "bedside" remains. We all make mistakes but if we don't understand why they occurred it is likely that we will repeat them.

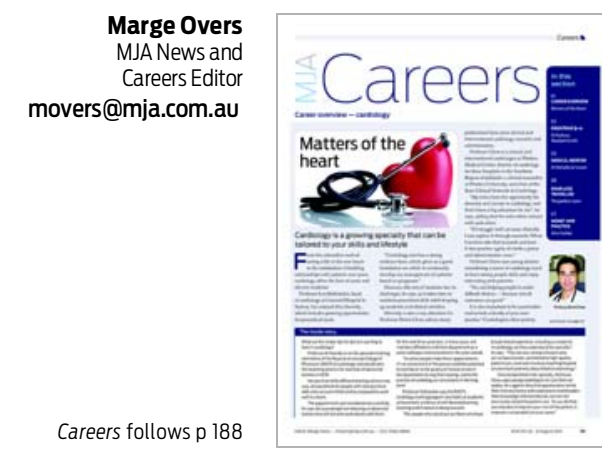

\section{Matters of the heart}

There are growing opportunities for young doctors considering a career in cardiology, say leading cardiologists quoted in this issue's Career Overview, which looks at the mix of acute, chronic and procedural medicine on offer in the specialty (page C1). MJA Careers also profiles WA cardiologist, Dr Michelle Ammerer, who explains what it takes to make it in the often stressful world of interventional cardiology (page C5). In Road Less Travelled, Melbourne anaesthetist and president of the Australian Doctors Orchestra, Dr Rowan Thomas, shares his love of music and medicine (page C6), and our Money and Practice section looks at the potential of social media tools, such as Twitter and Facebook, to link doctors with their profession (page C7) and other health professionals. 